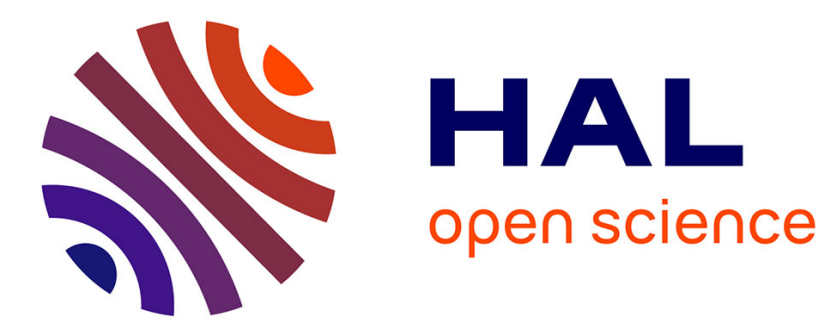

\title{
The costs and benefits of temporal predictability: impaired inhibition of prepotent responses accompanies increased activation of task-relevant responses
}

Inga Korolczuk, Boris Burle, Jennifer T Coull

\section{- To cite this version:}

Inga Korolczuk, Boris Burle, Jennifer T Coull. The costs and benefits of temporal predictability: impaired inhibition of prepotent responses accompanies increased activation of task-relevant responses. Cognition, 2018, 179, pp.102 - 110. 10.1016/j.cognition.2018.06.006 . hal-01848094v2

\section{HAL Id: hal-01848094 \\ https://hal.science/hal-01848094v2}

Submitted on 29 Apr 2019

HAL is a multi-disciplinary open access archive for the deposit and dissemination of scientific research documents, whether they are published or not. The documents may come from teaching and research institutions in France or abroad, or from public or private research centers.
L'archive ouverte pluridisciplinaire HAL, est destinée au dépôt et à la diffusion de documents scientifiques de niveau recherche, publiés ou non, émanant des établissements d'enseignement et de recherche français ou étrangers, des laboratoires publics ou privés. 


\title{
The costs and benefits of temporal predictability: impaired inhibition of prepotent responses accompanies increased activation of task-relevant responses th
}

\author{
Inga Korolczuk ${ }^{\mathrm{a}, *}$, Boris Burle ${ }^{\mathrm{b}}$, Jennifer T. Coull ${ }^{\mathrm{b}}$ \\ ${ }^{a}$ Institute of Applied Psychology, Jagiellonian University, ul. Lojasiewicza 4, 30-348 Krakow, Poland \\ ${ }^{b}$ Laboratoire des Neurosciences Cognitives UMR 7291, Federation 3C, Aix-Marseille University $\mathcal{G}$ CNRS, 3 Place Victor Hugo, 13331 Marseille cedex 3, France
}

\begin{abstract}
While the benefit of temporal predictability on sensorimotor processing is well established, it is still unknown whether this is due to efficient execution of an appropriate response and/or inhibition of an inappropriate one. To answer this question, we examined the effects of temporal predictability in tasks that required selective (Simon task) or global (Stop-signal task) inhibitory control of prepotent responses. We manipulated temporal expectation by presenting cues that either predicted (temporal cues) or not (neutral cues) when the target would appear. In the Simon task, performance was better when target location (left/right) was compatible with the hand of response and performance was improved further still if targets were temporally cued. However, Conditional Accuracy Functions revealed that temporal predictability selectively increased the number of fast, impulsive errors. Temporal cueing had no effect on selective response inhibition, as measured by the dynamics of the interference effect (delta plots) in the Simon task. By contrast, in the Stop-signal task, Stop-signal reaction time, a covert measure of a more global form of response inhibition, was significantly longer in temporally predictive trials. Therefore, when the time of target onset could be predicted in advance, it was harder to stop the impulse to respond to the target. Collectively, our results indicate that temporal cueing compounded the interfering effects of a prepotent response on task performance. We suggest that although temporal predictability enhances activation of task-relevant responses, it impairs inhibition of prepotent responses.
\end{abstract}

Keywords: temporal prediction, temporal preparation, timing, attention, response inhibition, response conflict

\section{Introduction}

Efficient adaptation to a complex environment requires not only that appropriate responses are selected and unwanted ones prevented, but also that these responses (or lack thereof) occur at appropriate moments in time. The ability to select precise moments in time in order to optimise behaviour depends upon the ability to make temporal predictions. Studies have shown that using abstract, yet temporally informative, cues to predict when an event will occur - a phenomenon known as the temporal orienting of attention - enhances sensorimotor processing of the event by improving accuracy (Correa, Lupiáñez, \& Tudela, 2005; Davranche, Nazarian, Vidal, \& Coull, 2011; Martens \& Johnson, 2005; Visser, 2014) and speeding response times (Coull \& Nobre, 1998; Nobre, 2001; Correa, Lupiáñez, \& Tudela, 2006). It is unknown, however, whether the beneficial effect of temporal cues on response time is due to more efficient selection of a response appropriate to the target and/or better inhibition of an inappropriate one. The goal of the present study was to examine the effects of temporal orienting on these two complementary aspects of motor control. These control processes have traditionally been investigated with so-called "conflict" tasks, such as the Simon (Simon, 1969), Flanker (Eriksen \& Eriksen, 1974) or Stroop (Stroop, 1935) tasks. In such tasks, stimuli are composed of two perceptual dimensions: one is relevant for the task at hand and defines the to-be-given response (for example a plus or a cross associated with a left or

\footnotetext{
This is the author's (postprint) version of the article published in Cognition, 179 (2018) 102-110

*E-mail address:inga.korolczuk@uj.edu.pl (I. Korolczuk). DOI:10.1016/j.cognition.2018.06.006, (2) the authors
}

right hand response, respectively); the second dimension, although irrelevant for the task, shares conceptual properties with the relevant dimension and/or response, and hence interferes with task goals (in the Simon task for example, the plus sign could be presented on the left side of the screen, compatible with the correct response, or on the right side, incompatible with it). Typically, reaction times to incompatible targets are slower than those to compatible ones, and this behavioural cost can be used to index the interference effect of response conflict. Recently, Menceloglu, Grabowecky and Suzuki (2017) failed to find an effect of temporal cueing on response conflict in the Flanker task. However, using both Flanker and Simon tasks, Correa, Cappucci, Nobre, and Lupiáñez (2010) found that temporal cueing significantly exacerbated the behavioural cost of response conflict. Specifically, the interference effect was even greater when participants were expecting the target to occur at a particular moment in time. The authors suggested that temporal orienting increased motor readiness for all targets, thereby facilitating correct responses on compatible trials but increasing interference on incompatible trials (see also Weinbach and Henik, 2013).

This effect could, however, stem from any one of the different processes that are needed to make a correct response. First, intention-guided action selection allows the appropriate goaldirected response to be deployed according to task instructions (Vohs, Baumeister, 2004). For example, a left-handed response can be activated after a presentation of a '+'. At the same time however, strong extraneous stimulus-action associations (for example, activation of a left-handed response to presenta- 
tion of a target on the left side of the screen) might activate inappropriate actions, through a process called response capture, which is fast and automatic in nature. In conflict tasks, the relevant dimension (e.g., the shape of the target in the example above) causes the intention-guided response to be activated, while the irrelevant one (target position) automatically triggers a stimulus-action association, which can be either compatible or incompatible with the intention-guided response. Since the intentional component of the selection process is thought to take time to build up (Ridderinkhof, 2002), fast responses are more likely to have been driven by prepotent stimulus-action associations. The relative strength and the time course of these processes can be estimated by Conditional Accuracy Functions (CAF), which plot the probability of making a correct response as a function of response speed. In conflict tasks, CAF usually reveal that fast responses to incompatible targets are more error-prone, indicating that fast action selection is driven more by strong, extraneous stimulus-action associations than by deliberate intentions (Ridderinkhof, Forstmann, Wylie, Burle, \& van den Wildenberg, 2011).

Another crucial mechanism in action control therefore, is response inhibition - active suppression of the inappropriate action. It can be engaged to suppress inappropriate responses (incorrect or premature) in favour of more goal-directed ones, or to suppress any action in general (Mostofsky \& Simmonds, 2008; Ridderinkhof et al., 2011). Two of the most predominantly used experimental paradigms for investigating response inhibition are the Simon task (Simon 1969; Simon 1990) and the Stop-signal task (Logan, Cowan, \& Davis, 1984; Verbruggen \& Logan, 2008). In the Simon task, selective inhibition is indexed by successful suppression of the response triggered by the task-irrelevant feature in favour of the response associated with the task-relevant feature. In the example given above, a ' ', presented on the right of the screen would require selective inhibition of the right-hand response, allowing the left-hand response to be deployed. It has been proposed that the dynamics of such suppression can be revealed in "delta-plots" (Ridderinkhof, 2002), which depict the magnitude of the interfering effect of the task-irrelevant feature as a function of reaction time. Specifically, slower response times show less of an interference effect than faster ones, because the inhibition process has had sufficient time to take effect. Accordingly, the greater the difference in the size of the interference effect between fast and slow response times (i.e., a more negative-going slope) the greater the influence of inhibition on performance.

By contrast to this selective inhibition of an inappropriate response in the Simon task, the Stop-signal task requires a more global form of inhibition. The Stop-signal paradigm involves two concurrent tasks, termed a go task, which is usually a discrimination task, and a stop task occurring on $25 \%$ of trials. During stop trials, an auditory tone is presented, which informs the subjects to refrain completely from giving their response on that trial. Performance on the task has been suggested to depend upon a race between two independent processes: the go process and the stopping process (Logan, 1994; Logan et al., 1984). If the stop process is faster than the go process, the response is successfully inhibited (i.e., no response is emitted). Conversely, if the go process is faster than the stop process then a response is incorrectly produced. Importantly, this race model allows the time taken to inhibit a response to be inferred, which is often termed the "Stop-signal reaction time" (SSRT).

The aim of our study was to measure the effects of temporal cueing on response activation and inhibition using both the Simon task (see also Correa et al., 2010) and, for the first time, the Stop-signal task. The use of both tasks allowed us to compare the effects of temporal predictability on response inhibition processes that were implemented either to selectively suppress erroneous responses to irrelevant stimulus-driven associations (Simon task) or to withhold responses entirely (Stopsignal task). Many previous studies of response inhibition have shown that presentation of non-specific warning cues in the Flanker paradigm increases interference effects, due either to a deleterious effect on cognitive control (e.g., Callejas, Lupiàñez, Funes, \& Tudela, 2005) or to enhanced sensory processing of irrelevant, as well as relevant, stimuli (e.g., Nieuwenhuis \& de Kleijn, 2013; Weinbach \& Henik, 2012b). Indeed, enhanced sensory processing has also been used to explain the beneficial effects of warning cues in the Stop-signal paradigm (Weinbach, Kalanthroff, Avnit, \& Henik, 2015). These authors have also made a clear distinction between warning cues (a non-specific state of alertness before target onset) and temporal cues (prediction of target onset) (Weinbach \& Henik, 2012a, 2013), and suggested that each might influence processing in similar, yet independent, ways. We extend this literature by measuring the effects of cues carrying temporally precise information on processes of response inhibition. We also aimed to refine the results of previous studies by using sensitive chronometric measures (CAF, delta plots) to more fully characterize the effects of temporal predictability on action control in terms of both response activation and response inhibition.

Finally, in contrast to previous studies (Correa et al., 2010, Menceloglu et al., 2017) in which the length of the delay between the cue and the target (the "foreperiod" [FP]) was manipulated in a blockwise fashion (equivalent to a fixed FP paradigm), we investigated the effects of trial-by-trial temporal cueing. In our variable FP paradigm, temporal cues predicted whether the target would appear after either a short or long FP, allowing the temporal focus of attention to be flexibly oriented from one trial to another within a block. In the control condition, targets also appeared after either short or long FPs but uninformative "neutral" cues did not predict the duration of the upcoming FP. This control condition not only allowed us to measure the performance benefits of temporal versus neutral cues, but also allowed us to measure more implicit forms of temporal expectation induced by the variable length of the FP itself. Typically, in a neutrally cued variable FP paradigm, response times are faster to targets presented after long, rather than short, FPs ("variable FP effect") or to targets presented after a FP that is identical to that of the preceding trial ("sequential effects" of FP) (Niemi \& Naatanen, 1981). Participants appear to automatically form temporal predictions about FP length based on the temporal statistics inherent in the trial or task structure (Los, Kruijne, \& Meeter, 2014) in order to speed responses. Different mechanisms have been proposed to account for observed data. For example, in the multiple trace theory, the memory trace of the FP encountered in more recent trials is stronger than that encountered in more distance ones and so contributes more to current behaviour (Los et al., 2014). Alternatively, in 
the dual-process model, hazard-based preparation is combined with automatic carryover of a refractory cost (Vallesi \& Shallice, 2007). Regardless of the theoretical account, results have revealed that sequential effects remain more resistant to different experimental manipulation such as dual-task interference and spatial context (Vallesi, Arbula, \& Bernardis, 2014; Los, 2004, respectively). Collectively, results suggest that sequential effects are driven by automatic processes, whereas the variable FP effect may be underpinned by more controlled processes. Based on previous findings, we formulated two hypotheses. If temporal cueing affects action control by increasing activation of correct responses (Correa et al., 2010), we would expect to see RT benefits in compatible, but not incompatible, trials of the Simon task, and in go trials of the Stop-signal task. More interestingly, we would predict specific effects on the CAF analysis of the Simon task, with temporal cueing reducing accuracy of very fast responses to incompatible targets. Alternatively, if temporal cueing affects action control by increasing inhibition of inappropriate responses (i.e. incorrect responses in the Simon task or premature responses in the Stop-signal task), its effects should be seen as a steeper slope in the delta plots of the Simon task, and as faster stopping times in the Stop-signal task.

\section{Method}

\subsection{Participants}

Forty-one undergraduate students ( 29 women, 12 men) took part in the study. Mean age was 20.8 years $(S D=2.0$, range 19-27). Ethics approval was obtained from the local research ethics committee (KEBN, Krakow, Poland). In the Simon task, data from six participants were discarded due to high error and omission rates ( \pm 2 SD of the group average) and/or an inconsistent finger-response mapping. Similarly, in the Stop-signal task, data from four participants were excluded due to high omission rates in go trials ( $\pm 2 \mathrm{SD}$ of the group average) and a considerably lower mean p(respond | signal) $( \pm 2 \mathrm{SD}$ of the group average), indicating that subjects inhibited substantially more than $50 \%$ of the time. The final sample was thus composed of 31 participants.

\subsection{Experimental tasks}

All participants performed temporally cued versions of two classic response inhibition tasks: the Simon task and the Stopsignal task. To facilitate interpretation of between-task comparisons, and to simplify instructions for the participants, we used comparable stimuli and timings across tasks (Fig. 1). The order of tasks was randomised. Tasks were presented using E-Prime software.

\subsubsection{Simon task}

The basic visual display consisted of a white centrally located cue $\left(1^{\circ}\right.$ eccentricity) presented on a black background (Fig. 1). Targets were white $1^{\circ}$ stimuli ("+" or " $\times$ "), which appeared either on the left or right side of the central cue at a distance of approximately $3^{\circ}$ of visual angle. The central cue was a stimulus consisting of two concentric circles, which provided information about when the target would appear. There were two cue conditions: temporal and neutral. In the temporal cue condition $(\mathrm{T})$, a brightening of the inner, smaller circle informed participants that a target would appear soon $(600 \mathrm{~ms})$, whereas a brightening of the outer, larger circle informed participants that a target would appear later $(1,400 \mathrm{~ms})$. All temporal cues were valid. In the neutral cue condition $(\mathrm{N})$, both the inner and outer circles were brightened providing no temporally precise information. Participants were asked to use the information provided (or not) by the cue to respond as quickly and accurately as possible according to the shape ("+" or " $X$ ") of the target. Half of the participants pressed 'a' on a standard QWERTY keyboard with their left index finger for the " + " and ' $\mathrm{l}$ ' with their right index finger for the " $x$ ". These finger-response pairings were reversed for the remaining participants. Although irrelevant for the task at hand, each target could be presented on either the left or right side of the screen. As a consequence, the lateralised response required by the target (left/right hand) could be on either the same (compatible condition) or the opposite (incompatible condition) side of target presentation. At the start of a trial, the cue ( $\mathrm{T}$ or $\mathrm{N}$ ) appeared for 500ms after which the background visual display was presented for one of the two FPs $(600 \mathrm{~ms}$ or $1,400 \mathrm{~ms})$. Next, the target appeared for $100 \mathrm{~ms}$, followed by the presentation of the background visual stimulus for $900 \mathrm{~ms}$, during which participants gave their response. The trial ended with the background display presented for a period of $500,600,700,800$ or $900 \mathrm{~ms}$ (i.e., the inter-trial interval), randomised across trials.

The two cue conditions ( $\mathrm{T}$ and $\mathrm{N}$ ) were presented in separate blocks. There were four blocks per cue condition, presented in randomised order. Each block contained 64 trials, resulting in 512 trials altogether. In each block, the proportion of compatible to incompatible trials was 50:50 and the proportion of short $(600 \mathrm{~ms})$ and long $(1,400 \mathrm{~ms})$ FPs was also 50:50. Both compatibility and FP conditions were randomised within a block. Across all blocks, this resulted in 64 trials for each of the 8 combinations of the cue, FP and compatibility conditions. Each block lasted approximately 3 to 4 minutes. Breaks between blocks were given. A training session was provided during which participants performed 60 trials to familiarise them with the task.

\subsubsection{Stop-signal task}

As in the Simon task, the basic visual display consisted of a white centrally located cue $\left(1^{\circ}\right.$ eccentricity) presented on a black background (Fig. 1), which provided information about when the target would appear. Again, targets were white $1^{\circ}$ stimuli ("+" or " $\times$ ") but, in contrast to the Simon task, they were not lateralised but appeared within the central cue.

As in the Simon task, there were two cue conditions: temporal and neutral. In the temporal cue condition ( $\mathrm{T})$, brightening of the inner, smaller circle informed participants that a target would appear soon $(600 \mathrm{~ms})$ and brightening of the outer, larger circle informed participants that a target would appear later $(1,400 \mathrm{~ms})$. Again, all temporal cues were valid. In the neutral cue condition $(\mathrm{N})$, both the inner and outer circles were brightened thus providing no temporally precise information. Participants were asked to use the temporal information the cue provided, to discriminate as quickly and accurately as possible the shape of the target ("+"/“×"). Half of the participants pressed ' $a$ ' on the manual keyboard with their left index finger for the " + " and ' 1 ' on the manual keyboard with their right in- 


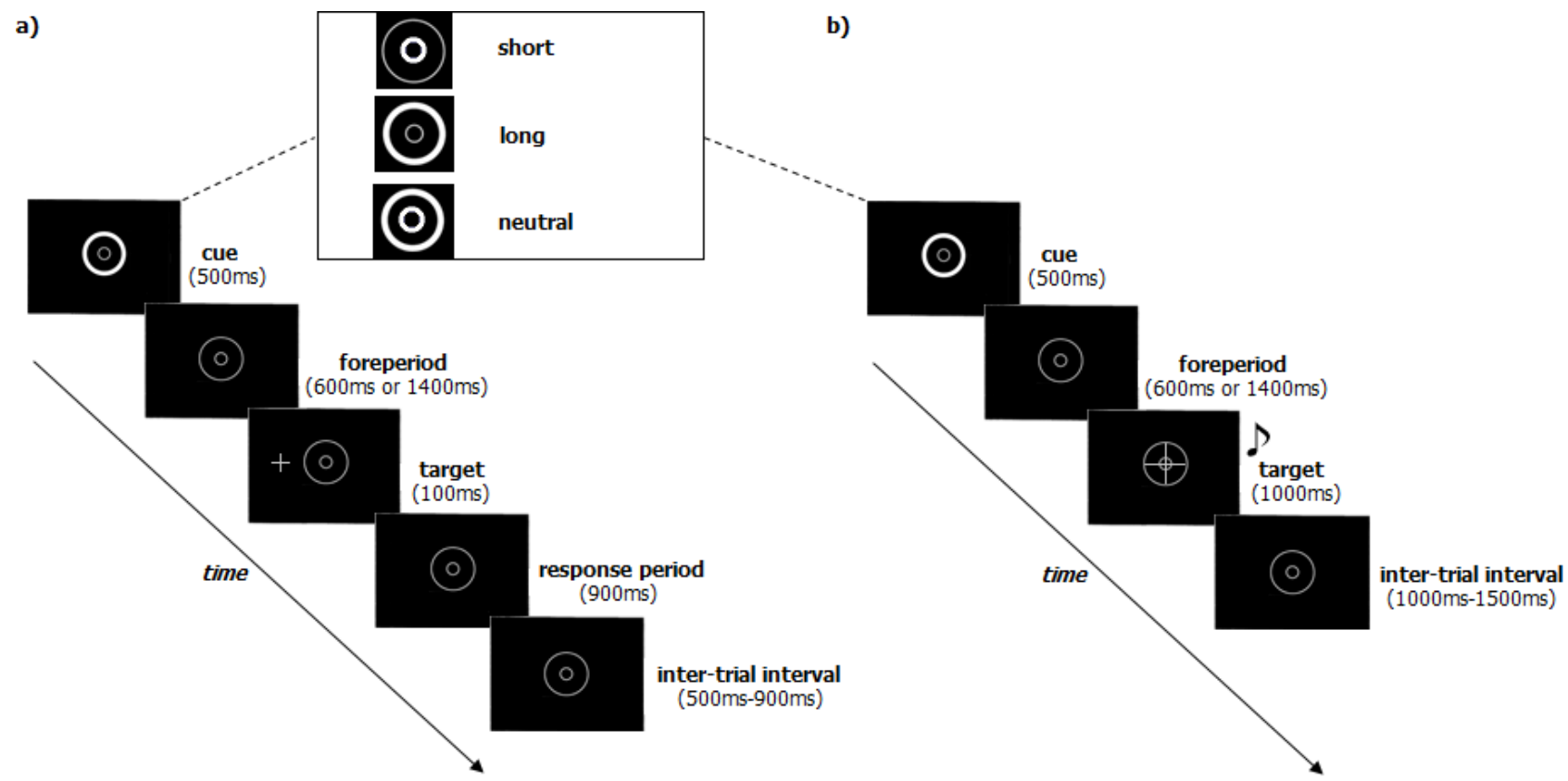

Figure 1: a. The trial timeline in the Simon task. A cue appeared for 500ms, providing (or not) temporal information as to when a target would occur. A background display was then presented for $600 \mathrm{~ms}$ or $1,400 \mathrm{~ms}$ (FP). Next, a target ("+" or " $\times$ ") appeared for 100ms on either the left or right side of the display, followed by a $900 \mathrm{~ms}$ period of the background display, during which participants made their choice response (left or right index finger for + or $\times$, counterbalanced across participants). The inter-trial interval was randomised between $500 \mathrm{~ms}-900 \mathrm{~ms}$. b. The trial timeline in the Stop-signal task. A cue was presented for 500ms informing (or not) as to the time of target occurrence. Then, after $600 \mathrm{~ms}$ or $1,400 \mathrm{~ms}(\mathrm{FP})$, the target ("+" or " $\times$ ") appeared in the centre of the display for $1,000 \mathrm{~ms}$, which was the time allowed for the choice response to be given (left or right index finger for + or $\times$, counterbalanced across participants). In $25 \%$ of trials, a sound was presented after variable interval (SSD) following target onset, meaning that participants had to withhold their response. The inter-trial interval was randomised between $1,000 \mathrm{~ms}-1,500 \mathrm{~ms}$

dex finger for the " $x$ " (the primary task). These finger-response pairings were reversed for the remaining participants. In $25 \%$ of the trials (stop trials), the target ("+" or " $\times$ ") was followed by an auditory stop signal (at variable delays, see below), instructing the participants that they should withhold their response (Logan et al., 1984).

At the start of the trial, the cue (T or N) appeared for 500ms followed by the background visual display presented for one of the two FPs $(600 \mathrm{~ms}$ or $1,400 \mathrm{~ms})$. Next, the target appeared within the circles and was presented for a duration of $1,000 \mathrm{~ms}$, irrespective of the participant's RT, and was the maximum time allowed for the response. In stop trials, the auditory stop signal $(750 \mathrm{~Hz}, 75 \mathrm{~ms})$ was then presented after a variable Stop-signal delay (SSD). The SSD was initially set at $250 \mathrm{~ms}$ and was adjusted continuously using a staircase procedure (Verbruggen, Logan, \& Stevens, 2008): if the participant successfully inhibited their response, the SSD increased by $50 \mathrm{~ms}$ on the next stop trial (i.e., a longer delay between target presentation and the auditory stop signal); if, however, the participant failed to inhibit their response, the SSD decreased by $50 \mathrm{~ms}$ on the next stop trial (i.e., a shorter delay). The trial ended with presentation of the background visual stimuli for a jittered interval of 1,000, $1,100,1,200,1,300,1,400$ or 1,500ms (i.e., the inter-trial interval). Two cue conditions ( $\mathrm{T}$ and $\mathrm{N}$ ) were presented in separate, randomised blocks. Again, there were four blocks per cue condition, with 64 trials per block, resulting in 512 trials altogether. In each block, the proportion of go to stop trials was 75:25 and the proportion of short $(600 \mathrm{~ms})$ to long $(1,400 \mathrm{~ms})$ FPs was 50:50. Both go/stop and FP conditions were randomised within a block. Altogether, this resulted in 96 trials for each of the 4

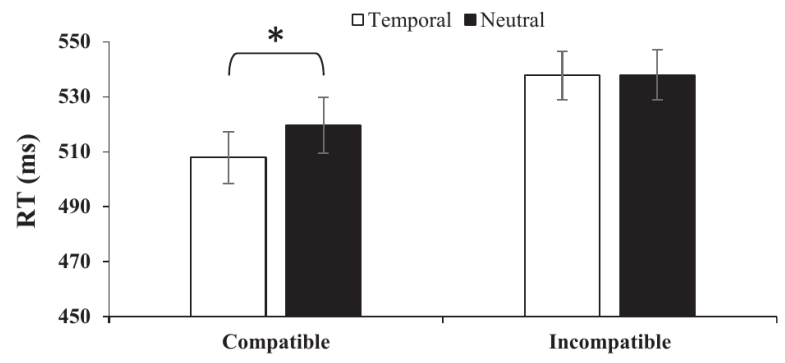

Figure 2: In the compatible condition, RTs were faster in the temporal condition as compared to the neutral condition. By contrast, in the incompatible condition the effect of temporal cueing disappeared, yielding no RT benefits of temporal cueing. Error bars reflect standard errors.

go combinations of the cue and FP conditions, and 32 trials for each of the 4 stop combinations of cue and FP conditions.

Each block lasted approximately 3 to 4 minutes. Breaks between blocks were given. A training session was provided during which participants performed 60 trials to familiarize them with the task.

\subsection{Data analysis}

\subsubsection{Simon task}

The mean response time (RT) in ms was calculated separately for the temporal and neutral conditions at the $600 \mathrm{~ms}$ and 1,400ms FPs and for compatible and incompatible conditions. We included data only from trials in which participants responded correctly. The rate of omission (missing responses) was approximately $3 \%$. To measure the effect of temporal pre- 
dictability on RT, a three-way repeated measures ANOVA involving cue (temporal, neutral), FP (short, long) and compatibility (compatible and incompatible) was performed. In order to measure variable FP (FP) and sequential effects on RT, a three-way repeated measures ANOVA involving FP of the current trial FP(n) (short, long), FP of the previous trial FP(n1) (short, long) and compatibility (compatible, incompatible) was conducted for neutral trial data only. To measure the effect of temporal predictability on the dynamics of the interference effect (incompatible - compatible RT) distribution analyses were performed. First, we "vincentized" (De Jong, Liang, \& Lauber, 1994; Ratcliff, 1979; Vincent, 1912) RTs from correct trials only, using a customized python script. This was done separately for each participant, and for each of the four temporal conditions (temporal/neutral cue; short/long FP) and the two compatibility conditions (compatible/incompatible targets). For each participant RTs were ranked in ascending order and binned into 5 classes (quintiles), each containing the same number of trials. The mean of each quintile was calculated. Then, for each quintile, the difference in mean RT between incompatible and compatible trials was extracted and used as the dependent variable. Plotting these values as a function of the mean RT of the quintile (i.e., the mean of both compatible and incompatible trials) has been referred to as a "delta-plot" (Pratte, Rouder, Morey, \& Feng, 2010; Ridderinkhof, 2002; Ridderinkhof, van den Wildenberg, Wijnen, \& Burle, 2004). A three-way repeated measures ANOVA involving cue (temporal, neutral), FP (short, long) and quintile (1 to 5) was performed on these delta values.

Accuracy (\% errors) was initially analysed using a threeway repeated measures ANOVA involving cue (temporal, neutral), FP (short, long) and compatibility (compatible and incompatible). To assess accuracy in a more dynamic way, Conditional Accuracy Functions (CAF) were computed for each participant and for each of the four temporal conditions and the two compatibility conditions. CAFs are also based on a vincentization of the data, though with some differences. Instead of focusing only on correct responses, all RTs, for both correct and error trials, were ranked in ascending order. Then, the percentage of correct responses within each quintile was computed and used as the dependent variable. A four-way repeated measures ANOVA involving cue (temporal, neutral), FP (short, long), compatibility (compatible, incompatible) and quintile (1 to 5) was performed to measure the effect of temporal predictability on the probability of correct responding as a function of RT. Bonferroni adjustments were made for pairwise comparisons. Effect sizes were calculated by using the partial eta-squared $\left(\eta_{\mathrm{p}}^{2}\right)$

\subsubsection{Stop-signal task}

The mean response time (RT) in ms for go trials was calculated separately for the temporal and neutral conditions at the $600 \mathrm{~ms}$ and 1,400ms FPs. We included data only from trials in which participants responded correctly. The rate of omission (missing responses) was approximately $2 \%$. The Stopsignal delay (SSD) was calculated as the mean delay between visual and auditory stimuli in both correct and incorrect stop trials. This was done separately for the temporal and neutral conditions and at the $600 \mathrm{~ms}$ and $1,400 \mathrm{~ms}$ FPs. In this proto- col, the Stop-signal RT (SSRT) corresponds to the mean time to inhibit a prepared response. SSRT cannot be measured directly and must instead be estimated. It was calculated by using the quantile method, which does not require the assumption of 50\% inhibition (Logan, 1994). With this method, the probability of responding to a stop signal [p(respond-signal)] is estimated for each participant. The RTs from correct go trials are ranked in ascending order to approximate an RT cumulative density function. The RT whose probability corresponds to $\mathrm{p}$ (respond-signal) is then chosen by multiplying the number of all reaction times in a given distribution by the probability of responding to a stop signal at a given delay [p(respond-signal)]. Subtracting SSD from this critical RT produces the SSRT.

To measure the effect of temporal predictability on go trial RTs, a two-way repeated measures ANOVA involving cue (temporal, neutral) and FP (short, long) was performed. In order to determine the effect of temporal predictability on SSRT, a twoway repeated measures ANOVA using data from stop trials was conducted, comprising cue (temporal, neutral) and FP (short, long). Similarly, to determine the effect of temporal predictability on SSD, a two-way repeated measures ANOVA involving cue (temporal, neutral) and FP (short, long) was performed. To measure the effect of temporal predictability on Signal-respond RT (SRRT), which is the RT of incorrectly emitted responses in stop trials, a two-way repeated measures ANOVA with cue (temporal, neutral) and FP (short, long) was conducted.

Overall accuracy (\% errors) was analysed in go trials using a two-way repeated measures ANOVA involving cue (temporal, neutral) and FP (short, long). Finally, the mean percentage of the failure to inhibit a response in stop trials [p(respond-signal)], was analysed using a two-way repeated measures ANOVA involving cue (temporal, neutral) and FP (short, long).

\section{Results}

\subsection{Simon task \\ 3.1.1. Temporal cueing}

To measure the effect of temporal cueing on RT, a three-way repeated measures ANOVA involving cue (temporal, neutral), FP (short, long) and compatibility (compatible and incompatible) was performed. There was a main effect of compatibility on RT, $F(1,30)=45.19, p<.001, \eta_{\mathrm{p}}^{2}=.60$, with slower RTs for incompatible versus compatible targets. There was no main effect of cue, $F(1,30)=2.31, p=.139, \eta_{\mathrm{p}}^{2}=.07$, or FP, $F(1,30)=2.64, p=.115, \eta_{\mathrm{p}}^{2}=.08$ on RT, nor a Cue $\times$ FP interaction, $F(1,30)=3.87, p=.058, \eta_{\mathrm{p}}^{2}=.11$, or an $\mathrm{FP} \times \mathrm{Com}-$ patibility interaction, $F(1,30)=0.001, p=.980, \eta_{\mathrm{p}}^{2}<.001$. Importantly, however, the results showed a significant Cue $\times$ Compatibility interaction, $F(1,30)=5.29, p=.029, \eta_{p}^{2}=.15$. This interaction was further broken down by compatibility (Fig. 2). For compatible targets, there was a significant cue main effect with participants responding significantly faster in temporal cue trials than in neutral trials $(p=.044)$. However, there was no significant difference in RTs between temporal and neutral trials for incompatible targets $(p=.953)$. In parallel, RTs were slower for incompatible than compatible trials in both the temporal $(p<.001)$ and neutral $(p<.001)$ condition, though this interference effect was quantitatively greater in the temporal condition. 
Table 1: Mean (and standard error) reaction times (ms) and accuracy during the Simon task.

\begin{tabular}{|c|c|c|c|c|c|}
\hline \multirow[b]{2}{*}{ Compatibility } & \multirow[b]{2}{*}{ Foreperiod } & \multicolumn{2}{|l|}{ Reaction times } & \multicolumn{2}{|l|}{$\%$ Accuracy } \\
\hline & & Temporal cue & Neutral cue & Temporal cue & Neutral cue \\
\hline \multirow[t]{2}{*}{ Compatible } & Short & $507(9)$ & $524(10)$ & $93.06(17)$ & $92.84(17)$ \\
\hline & Long & $509(10)$ & $515(11)$ & 93.77 (17) & 95.01 (17) \\
\hline \multirow[t]{2}{*}{ Incompatible } & Short & $538(9)$ & $540(10)$ & $86.63(16)$ & $89.92(17)$ \\
\hline & Long & $537(9)$ & $535(9)$ & 87.85 (16) & 90.47 (17) \\
\hline
\end{tabular}

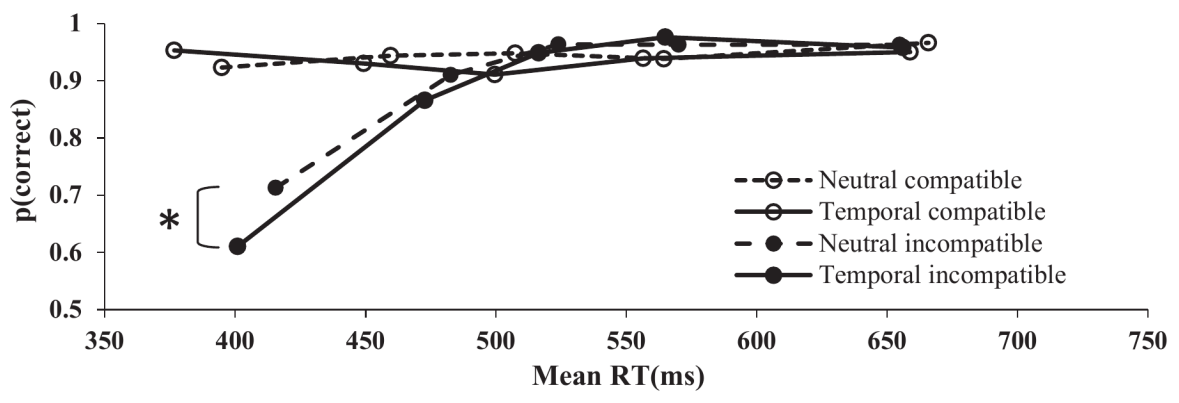

Figure 3: Conditional accuracy functions (CAF). The plots show the probability of correct response as a function of RT in both temporal and neutral conditions for compatible and incompatible targets. For incompatible targets (black circles), participants made more fast errors in temporal (solid line) as compared to neutral (dashed line) condition.

To measure the effect of temporal cueing on accuracy ( $\%$ errors), data were analysed using a three-way repeated measures ANOVA involving cue (temporal, neutral), FP (short, long) and compatibility (compatible and incompatible). The analysis of accuracy rates revealed a significant compatibility main effect, $F(1,30)=21.55, p<.001, \eta_{\mathrm{p}}^{2}=.42$. As expected, participants were less accurate for incompatible than compatible targets. We also found a cue main effect, $F(1,30)=4.36, p=.045, \eta_{\mathrm{p}}^{2}=$ .13 , with less accurate responses in temporal than neutral trials, and a main effect of FP, $F(1,30)=9.03, p=.005, \eta_{\mathrm{p}}^{2}=.23$, with participants being less accurate at short rather than long FPs. No Cue $\times$ Compatibility, $F(1,30)=2.07, p=.161, \eta_{\mathrm{p}}^{2}=$ .06 , nor FP $\times$ Compatibility, $F(1,30)=0.27, p=.611, \eta_{\mathrm{p}}^{2}=$ .01 , interaction was observed.

\subsubsection{FP effects}

To measure effects of the more implicit forms of temporal predictability indexed by variable FP (FP) and sequential effects, a three-way repeated measures ANOVA involving FP of the current trial FP(n) (short, long), FP of the previous trial FP(n-1) (short, long) and compatibility (compatible, incompatible) was conducted on neutral trial RTs only. We found main effects of $\mathrm{FP}(\mathrm{n}), F(1,30)=5.23, p=.029, \eta_{\mathrm{p}}^{2}=.15, \mathrm{FP}(\mathrm{n}-$ $1), F(1,30)=19.08, p<.001, \eta_{\mathrm{p}}^{2}=.39$, and compatibility, $F(1,30)=18.57, p<.001, \eta_{\mathrm{p}}^{2}=.38$, on RT. There was a significant $\mathrm{FP}(\mathrm{n}) \times \mathrm{FP}(\mathrm{n}-1)$ interaction, $F(1,30)=11.08, p=$ $.002, \eta_{\mathrm{p}}^{2}=.27$. RTs were significantly faster when the current short $\mathrm{FP}(\mathrm{n})$ trial was preceded by a short $\mathrm{FP}(\mathrm{n}-1)$ trial than by a long $\mathrm{FP}(\mathrm{n}-1)$ trial $(p<.001)$. By contrast, RTs were not significantly different when the current long $\mathrm{FP}(\mathrm{n})$ trial was preceded by either the short $\mathrm{FP}(\mathrm{n}-1)$ trial or long $\mathrm{FP}(\mathrm{n}-1)$ trial $(p=.707)$. The findings demonstrated the typical pattern of sequential effects, with FP(n-1) influencing short FP trials only. However, no interaction between compatibility and $\mathrm{FP}(\mathrm{n}), F(1,30)=0.54$, $p=.470, \eta_{\mathrm{p}}^{2}=.02$, or $\mathrm{FP}(\mathrm{n}-1), F(1,30)=0.51, p=.480, \eta_{\mathrm{p}}^{2}=$ .02 , was found.

\subsubsection{Distribution analysis}

To explore the dynamics of the temporal predictability effect on response speed and accuracy, we created RT distribution profiles for each participant by ranking RTs and binning them into 5 quintiles (see Methods).

First, we plotted Conditional Accuracy Functions (CAF), which show accuracy rates as a function of RT (Fig. 3). To measure the effect of temporal predictability on the probability of correct responding as a function of RT a four-way repeated measures ANOVA involving cue (temporal, neutral), FP (short, long), compatibility (compatible, incompatible) and quintile (1 to 5) was performed. Replicating numerous reports, there were main effects of compatibility, $F(1,30)=23.37, p<.001, \eta_{\mathrm{p}}^{2}=$ .44 , FP, $F(1,30)=5.23, p=.029, \eta_{\mathrm{p}}^{2}=.15$, and quintile, $F(4,120)=59.73, p<.001, \eta_{\mathrm{p}}^{2}=.67$. Furthermore, the analysis revealed a Compatibility $\times$ Quintile interaction, $F(4,120)=$ $63.09, p<.001, \eta_{\mathrm{p}}^{2}=.68$; as expected, the accuracy was lower for incompatible than compatible targets in quintile $1(p<$ $.001)$, quintile $2(p=.013)$, quintile $3(p=.013)$ and quintile 4 $(p<.001)$ but not in quintile $5(p=.794)$. Importantly, a significant main effect of cue, $F(1,30)=4.67, p=.039, \eta_{\mathrm{p}}^{2}=.14$, was qualified by a significant Cue $\times$ Compatibility $\times$ Quintile interaction, $F(4,120)=5.00, p=.001, \eta_{p}^{2}=.14$. The accuracy of responding to incompatible targets was significantly lower in temporal versus neutral conditions for the fastest RTs only (quintile 1), $p=.030$.

To measure the effect of temporal predictability on the dynamics of the interference effect, a three-way repeated measures ANOVA involving cue (temporal, neutral), FP (short, long) and quintile (1 to 5) was performed on the difference (delta value) between incompatible and compatible RTs. The analysis of the interference effect as a function of RT (delta plots), revealed a significant main effect of quintile, $F(4,120)=56.66, p<$ $.001, \eta_{\mathrm{p}}^{2}=.65$, with, as expected, a reduction in the interference effect as a function of RT (Fig. 4). We also found a significant main effect of cue, $F(1,30)=5.29, p=.029, \eta_{\mathrm{p}}^{2}=.15$, with a larger interference effect in temporal versus neutral trials. However, there was no significant effect of $F P, F(1,30)=$ 


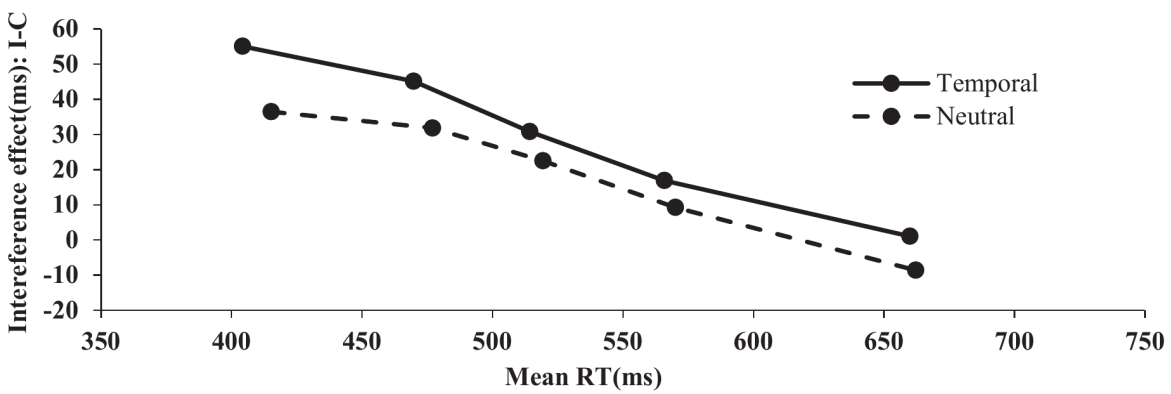

Figure 4: Delta plots. The plots show the interference effect (incompatible - compatible RT) as a function of RT for temporal and neutral conditions. The interference effect was greater in temporal condition (solid line) as compared to neutral condition (dashed line). The interference effect for both temporal and neutral conditions decreased as a function of RT.

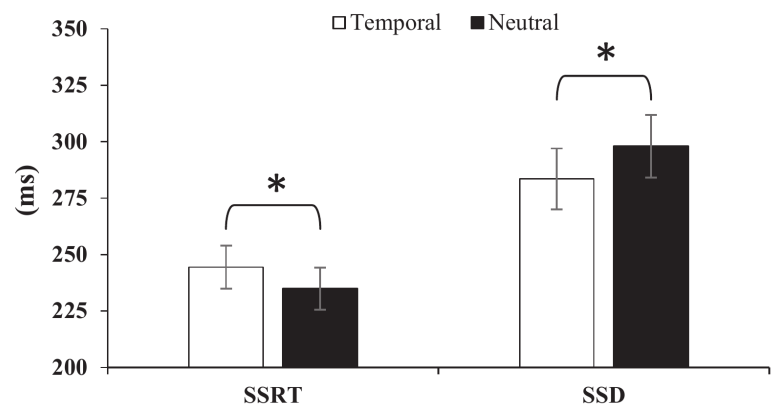

Figure 5: Mean SSRT and SSD in the Stop-signal task. In temporal trials, SSRT was significantly longer when compared to neutral trials. In turn, SSD was significantly shorter in temporal than neutral trials. Error bars reflect standard errors.

$0.001, p=.981, \eta_{\mathrm{p}}^{2}<.001$, nor, importantly, any significant interaction between quintile and cue, $F(4,120)=1.11, p=$ $.356, \eta_{\mathrm{p}}^{2}=.04$, or FP, $F(4,120)=0.28, p=.890, \eta_{\mathrm{p}}^{2}=.01$.

\subsection{Stop-signal task}

\subsubsection{Go trials}

To measure the effect of temporal predictability on go trial RTs, a two-way repeated measures ANOVA involving cue (temporal, neutral) and FP (short, long) was performed. There was a significant main effect of cue, with participants responding faster in temporal compared to neutral condition (i.e., temporal cueing effect), $F(1,30)=7.86, p=.009, \eta_{\mathrm{p}}^{2}=.21$. There was also a main effect of FP, $F(1,30)=4.81, p=.036, \eta_{\mathrm{p}}^{2}=$ .14 , which was further explained by a Cue $\times$ FP interaction, $F(1,30)=7.20, p=.012, \eta_{\mathrm{p}}^{2}=.19$. Post-hoc tests revealed that in the neutral condition, RTs were faster for targets presented at the long FP than at the short FP (i.e., variable FP effect) $(p=.005)$, but it was not the case in temporal condition $(p=.849)$. In parallel, there were faster RTs in the temporal versus neutral condition at the short FP $(p=.001)$, but not at the long FP ( $p=.308)$, confirming many previous results (e.g., Coull \& Nobre, 1998; Correa et al., 2005).

Overall accuracy (\% errors) in go trials was analysed using a two-way repeated measures ANOVA involving cue (temporal, neutral) and FP (short, long). There was no significant main effect of cue, $F(1,30)=1.73, p=.198, \eta_{\mathrm{p}}^{2}=.06$, nor Cue $\times$ FP interaction, $F(1,30)=0.37, p=.550, \eta_{\mathrm{p}}^{2}=.01$. However, a significant FP main effect, $F(1,30)=4.75, p=.037, \eta_{\mathrm{p}}^{2}=$ .14 , indicated that participants were less accurate in short FP $(97.13 \%)$ versus long FP $(97.68 \%)$ trials.
Table 2: Stop-signal task variables: Go RT, Stop-signal RT (SSRT), Stop-signal delay (SSD) and Signal-respond RT (SRRT) with standard errors (ms).

\begin{tabular}{llll}
\hline Stop-signal task variables & Foreperiod & Temporal cue & Neutral cue \\
\hline Go RT & Short & $550(12)$ & $566(12)$ \\
& Long & $550(12)$ & $554(13)$ \\
SSRT & Short & $247(10)$ & $236(9)$ \\
& Long & $242(10)$ & $234(11)$ \\
SSD & Short & $283(14)$ & $298(14)$ \\
& Long & $285(13)$ & $298(14)$ \\
SRRT & Short & $496(10)$ & $503(9)$ \\
& Long & $493(11)$ & $501(11)$ \\
\hline
\end{tabular}

\subsubsection{Stop trials}

To determine the effect of temporal cueing on the estimated RT to stop in stop trials, a two-way repeated measures ANOVA comprising cue (temporal, neutral) and FP (short, long) was conducted on the Stop-signal RT (SSRT) and the Stop-signal delay (SSD). Importantly, the analysis of SSRT revealed a significant main effect of cue, $F(1,30)=4.99, p=.033, \eta_{\mathrm{p}}^{2}=.14$. SSRTs were slower in temporal versus neutral trials. There was no main effect of FP, $F(1,30)=0.57, p=.458, \eta_{\mathrm{p}}^{2}=.02$, nor Cue $\times$ FP interaction, $F(1,30)=0.28, p=.868, \eta_{\mathrm{p}}^{2}=.001$, on SSRTs. Similarly, the analysis of SSD also showed a significant main effect of cue, $F(1,30)=11.13, p=.002, \eta_{\mathrm{p}}^{2}=.27$, with the SSD being shorter in temporal trials than in neutral trials. There was no main effect of FP on SSD, $F(1,30)=0.25, p=$ $.622, \eta_{\mathrm{p}}^{2}=.01$, nor Cue $\times$ FP interaction, $F(1,30)=0.20, p=$ $.658, \eta_{\mathrm{p}}^{2}=.01$. To measure the effect of temporal predictability on Signal-respond RT (SRRT), which is the RT of incorrectly emitted responses in stop trials, a two-way repeated measures ANOVA with cue (temporal, neutral) and FP (short, long) was conducted. This analysis failed to reveal any significant effects.

Finally, the mean percentage of the failure to inhibit a response in stop trials [p(respond-signal)], was analysed using a two-way repeated measures ANOVA involving cue (temporal, neutral) and FP (short, long). This analysis did not reveal any significant effects.

\section{Discussion}

Temporal prediction generally improves accuracy in "simple" target detection tasks. The origin of this improvement, however, is still unknown. In the present study, using conflict and inhibition tasks, we sought to determine whether this improvement is due to greater activation of a motor response appropriate to the target and/or better inhibition of an inappropriate one. The use of a Simon conflict task, coupled with advanced distribution analysis, allowed us to dissociate the strength 
of automatic response activation from its subsequent selective suppression. The Stop-signal task allowed us to measure the efficiency of a more global inhibitory process.

In the Simon task, temporal (versus neutral) cueing speeded response times to compatible targets, which is consistent with previous findings indicating that temporally predictive cue enhances response preparation (Correa \& Nobre, 2008; Miniussi, Wilding, Coull, \& Nobre, 1999; Nobre, 2001). By contrast, incompatible targets eliminated the RT benefits of temporal cueing. This dissociation suggests that knowing when to respond is only useful if there is no conflict about what to respond. Moreover, the interfering effect of response conflict was greater in the temporal versus neutral condition. Together, these results confirm the general pattern found by Correa et al. (2010) and indicate a mutual influence between temporal orienting and response compatibility.

To complement and extend the results of Correa et al. (2010), we analysed accuracy as well as response times. Although temporal cueing speeded response times to compatible targets, it induced more errors generally. To better understand the nature of this speed-accuracy trade-off, we plotted Conditional Accuracy Functions (CAF), which illustrate accuracy as a function of response time. Our data showed the typical pattern: there were more incorrect responses to incompatible targets when response times were fast (van den Wildenberg et al., 2010).

Importantly, this effect was exacerbated if participants had already formed a temporal expectation of when the target would occur. In other words, incompatible targets provoked a higher number of fast, erroneous responses in the temporal, compared to neutral, condition. These findings suggest that temporally predictable targets make participants more vulnerable to response capture by prepotent stimulus-response associations, thereby inducing a higher proportion of inappropriate responses.

Data from the Simon task further suggested that temporal orienting did not influence selective response inhibition. The slope of the delta plot at the slow end of the response time distribution, which indicates the effectiveness of suppressing the automatically activated response, was not influenced by temporal orienting, suggesting that selective inhibitory processes themselves were intact. Moreover, temporal cueing affected RTs in compatible trials only, not incompatible ones. This indicates an effect of temporal cueing primarily on response activation (compatible trials) rather than response inhibition (incompatible trials). Our Simon task results therefore appear to confirm the suggestion by Correa et al. (2010) that temporal orienting acts by increasing the overall level of response activation, rather than by affecting inhibition. However, in the Stop-signal task, which measures a more global form of response inhibition, Stop-signal reaction time (SSRT) was significantly slower in the temporal versus neutral condition. This result could be due either to faster SSRTs in the neutral condition (Weinbach et al., 2015) or slower SSRTs in the temporal condition. In parallel, response times to go targets were significantly faster in the temporal versus neutral condition. The complementary pattern of temporal cue effects on go and stop RTs indicates that when participants have a temporal expectation about the time of target onset it is easier to execute a response to the target, but it is also harder to stop it.

Data from the Stop-signal task suggest effects of temporal orienting on both response activation and inhibition. Yet selective response inhibition in the Simon task was unaffected by temporal orienting. These apparently contradictory results can be reconciled by dissociating the effects of temporal prediction on selective versus global inhibitory processes. Both formal modeling (Bausenhart, Rolke, Seibold, \& Ulrich, 2010) and electrophysiological data (Tandonnet et al., 2012) indicate that temporal predictability enhances activation, which, in turn, allows evidence accumulation to begin earlier. However, there is extensive evidence that during the warning period of an RT task, this activation is accompanied by a distinct inhibitory mechanism that keeps responses in check to ensure they are not emitted prematurely (Badry et al., 2009; Davey, Romaiguere, Maskill, \& Ellaway, 1994; Davranche et al., 2007; Duque, Lew, Mazzocchio, Olivier, \& Ivry, 2010; Greenhouse, Sias, Labruna, \& Ivry, 2015; Hasbroucq et al., 1999; Hasbroucq, Kaneko, Akamatsu, \& Possama1, 1997; Touge, Taylor, \& Rothwell, 1998). Our results may therefore reflect an effect of temporal orienting on inhibitory processes that guide the time of response (global inhibition), which prevents premature responding, rather than those guiding the type of response (selective inhibition), which prevents incorrect responding.

Indeed, inhibition plays a crucial role in temporal preparation (Correa, Triviño, Pérez-Dueñas, Acosta, \& Lupiáñez, 2010; Los, 2013) and the elapse of time itself might be coded by the degree of motor inhibition (Coull, Vidal, \& Burle, 2016; Kononowicz \& van Rijn, 2015). In our experiment, we suggest that temporal orienting both increased activation and reduced global inhibition (see also Davranche et al., 2007), thereby explaining both accelerated response times and the inability to stop prepotent responses. Alternatively, though not mutually exclusively, the fact that temporal cueing interferes with the inhibitory processes measured by the Stop-signal task, but not those measured by the Simon task, could be due to differences in the spatial certainty of target location in the two tasks. In the temporal condition of the Stop-signal task, participants know both when and where the target will appear. By contrast, in the Simon task, they know when it will appear but not where. Since temporal preparation is more effective when target location is known in advance (e.g., Doherty, Rao, Mesulam, \& Nobre, 2005; Rohenkohl, Gould, Pessoa, \& Nobre, 2014), temporal cueing effects might be stronger in the spatially certain Stop-signal task than the spatially uncertain Simon task. Nevertheless, in their study, Seibold and Rolke (2014a) failed to find effects of temporal preparation on spatial selection when task-irrelevant stimuli needed to be ignored, even when the target spatial position was predictable (although they found attentional advantage of temporal preparation in a visual search task with no distracting event, Seibold \& Rolke, 2014b). In the context of sensorimotor processing, our results indicate that temporal orienting lowers the response threshold, resulting in responses that are based on a lower quality of information. Even though temporal cueing led to faster RTs in compatible (Simon task) and go (Stop-signal task) trials, it also induced a more pronounced speed-accuracy trade-off in the Simon task and greater impulsivity in the Stop-signal task. It is important to note, however, that this pattern of results does not necessarily contradict previous findings that temporal preparation improves sensory processing in non-conflict tasks (Correa et al., 2005; Rolke, 
2008; Rolke \& Hofmann, 2007). It is plausible that the possibility of response conflict interferes with the benefits of temporal orienting on sensory processing that are normally found in perceptual identification tasks (Rolke, 2008; Vangkilde, Coull, \& Bundesen, 2012).

Finally, more implicit measures of temporal expectation indexed by variable FP (FP) and sequential effects were unaffected by response compatibility in the Simon task. These results indicate that temporal expectations formed implicitly by FP and sequential effects are more resistant to response conflict than those formed explicitly by temporal cues (Capizzi, Sanabria, \& Correa, 2012; Correa, Cona, Arbula, Vallesi, \& Bisiacchi, 2014). Moreover, the lack of compatibility effect suggests that FP and sequential effects influence sensorimotor processing after the response selection stage that is measured by response compatibility effects. In line with our interpretation of temporal cueing effects outlined above, FP and sequential effects might influence the global inhibition processes measured by the Stop-signal task ("should I make a response?") rather than the selective inhibition processes measured by the Simon task ("which response should I make?"). Unfortunately, FP and sequential effects could not be measured in the Stop-signal task because a single staircase tracking procedure was used for both FPs, which did not allow effects at short and long FPs to be disentangled. In the future, it would be informative to design experimental paradigms that allowed FP and sequential effects to be measured in Stop-signal tasks.

Taken together, the results of both the Simon and Stopsignal tasks revealed a consistent and complementary picture of the effect of temporal predictability on action control. In both tasks, a pre-formed temporal expectancy caused a prepotent externally triggered response to influence task performance by affecting both response activation and global inhibition. This mechanism would explain both the beneficial effects of temporal cues in simple and choice RT tasks (Coull \& Nobre, 1998; Correa et al., 2006) as well as the cost of temporal cueing when a prepotent response has to be inhibited (Correa et al., 2010).

\section{References}

Badry, R., Mima, T., Aso, T., Nakatsuka, M., Abe, M., Fathi, D., ... Fukuyama, H. (2009). Suppression of human cortico-motoneuronal excitability during the Stop-signal task. Clinical Neurophysiology, 120(9), 1717-1723. https://doi.org/10.1016/j.clinph.2009.06.027

Bausenhart, K. M., Rolke, B., Seibold, V. C., \& Ulrich, R. (2010). Temporal preparation influences the dynamics of information processing: Evidence for early onset of information accumulation. Vision Research, 50(11), 1025-1034. https://doi.org/10.1016/j.visres.2010.03.011

Callejas, A., Lupiàñez, J., Funes, M. J., \& Tudela, P. (2005). Modulations among the alerting, orienting and executive control networks. Experimental Brain Research, 167(1), 27-37. https://doi.org/10.1007/s00221005-2365-z

Capizzi, M., Sanabria, D., \& Correa, Á. (2012). Dissociating controlled from automatic processing in temporal preparation. Cognition, 123, 293-302. https://doi.org/10.1016/j.cognition.2012.02.005

Correa, Á., Cappucci, P., Nobre, A. C., \& Lupiáñez, J. (2010). The two sides of temporal orienting: Facilitating perceptual selection, disrupting response selection. Experimental Psychology, 57(2), 142-148. https://doi.org/10.1027/1618-3169/a000018

Correa, Á., Cona, G., Arbula, S., Vallesi, A., \& Bisiacchi, P. (2014). Neural dissociation of automatic and controlled temporal preparation by transcranial magnetic stimulation. Neuropsychologia, 65, 131-136. https://doi.org/ 10.1016/j.neuropsychologia.2014.10.023
Correa, Á., Lupiáñez, J., \& Tudela, P. (2005). Attentional preparation based on temporal expectancy modulates processing at the perceptual level. Psychonomic Bulletin \& Review, 12(2), 328-334. https://doi.org/10.3758/ BF03196380

Correa, Á., Lupianez, J., \& Tudela, P. (2006). The attentional mechanism of temporal orienting: Determinants and attributes. Experimental Brain Research, 169(1), 58-68. https://doi.org/10.1007/s00221-005-0131-x

Correa, Á., \& Nobre, A. C. (2008). Neural Modulation by Regularity and Passage of Time. Journal of Neurophysiology, 100(3), 1649-1655. https://doi.org/10.1152/jn.90656.2008

Correa, Á., Triviño, M., Pérez-Dueñas, C., Acosta, A., \& Lupiáñez, J. (2010). Temporal preparation, response inhibition and impulsivity. Brain and Cognition, 73(3), 222-228. https://doi.org/10.1016/j.bandc.2010.05.006

Coull, J. T., Vidal, F., \& Burle, B. (2016). When to act, or not to act: That's the SMA's question. Current Opinion in Behavioral Sciences, 8, 14-21. https://doi.org/10.1016/j.cobeha.2016.01.003

Coull, J. T., \& Nobre, A. C. (1998). Where and when to pay attention: the neural systems for directing attention to spatial locations and to time intervals as revealed by both PET and fMRI. Journal of Neuroscience, 18(18), 7426-7435. https://doi.org/0270-6474/98/187426-10\$05.00/0

Davey, N. J., Romaiguere, P., Maskill, D. W., \& Ellaway, P. H. (1994). Suppression of voluntary motor activity revealed using transcranial magnetic stimulation of the motor cortex in man. Journal of Physiology, 477(2), 223-235.

Davranche, K., Nazarian, B., Vidal, F., \& Coull, J. (2011). Orienting Attention in Time Activates Left Intraparietal Sulcus for Both Perceptual and Motor Task Goals. Journal of Cognitive Neuroscience, 23(11), 3318-3330. https://doi.org/10.1162/jocn_a_00030

Davranche, K., Tandonnet, C., Burle, B., Meynier, C., Vidal, F., \& Hasbroucq, T. (2007). The dual nature of time preparation: Neural activation and suppression revealed by transcranial magnetic stimulation of the motor cortex. European Journal of Neuroscience, 25(12), 3766-3774. https://doi.org/10.1111/j.1460-9568.2007.05588.x

De Jong, R., Liang, C. C., \& Lauber, E. (1994). Conditional and Unconditional Automaticity: A Dual-Process Model of Effects of Spatial Stimulus-Response Correspondence. Journal of Experimental Psychology: Human Perception and Performance, 20(4), 731-750. https://doi.org/10.1037/0096-1523.20.4.731

Doherty, J. R., Rao, A., Mesulam, M. M., \& Nobre, A. C. (2005). Synergistic Effect of Combined Temporal and Spatial Expectations on Visual Attention. Journal of Neuroscience, 25(36), 8259-8266. https://doi.org/10.1523/JNEUROSCI.1821-05.2005

Duque, J., Lew, D., Mazzocchio, R., Olivier, E., \& Ivry, R. B. (2010). Evidence for Two Concurrent Inhibitory Mechanisms during Response Preparation. Journal of Neuroscience, 30(10), 3793-3802. https://doi.org/10.1523/JNEUROSCI.5722-09.2010

Eriksen, B. A., \& Eriksen, C. W. (1974). Effects of noise letters upon the identification of a target letter in a nonsearch task. Perception and Psychophysics, 16(1), 143-149. http://dx.doi.org/10.3758/BF03203267

Greenhouse, I., Sias, A., Labruna, L., \& Ivry, R. B. (2015). Nonspecific Inhibition of the Motor System during Response Preparation. Journal of Neuroscience, 35(30), 10675-10684. https://doi.org/10.1523/JNEUROSCI.143615.2015

Hasbroucq, T., Kaneko, H., Akamatsu, M., \& Possama1, C.A. (1997). Preparatory inhibition of cortico-spinal excitability: a transcranial magnetic stimulation study in man. Cognitive Brain Research, 5(3), 185-192. https://doi.org/10.1016/S0926-6410(96)00069-9

Hasbroucq, T., Osman, A., Possamaï, C.A., Burle, B., Carron, S., Dépy, D., ... Mouret, I. (1999). Cortico-spinal inhibition reflects time but not event preparation: neural mechanisms of preparation dissociated by transcranial magnetic stimulation. Acta Psychologica, 101(2-3), 243-266. https://doi.org/10.1016/S0001-6918(99)00007-4

Kononowicz, T. W., \& van Rijn, H. (2015). Single trial beta oscillations index time estimation. Neuropsychologia, 75, 381-389. https://doi.org/10.1016/j.neuropsychologia.2015.06.014

Logan, G. D. (1994). On the ability to inhibit thought and action: A users guide to the stop-signal paradigm. Inhibitory Processes in Attention, Memory, and Language. https://doi.org/10.1016/j.jsat.2006.09.008

Logan, G. D., Cowan, W. B., \& Davis, K. A. (1984). On the Ability to Inhibit Simple and Choice Reaction Time Responses: A Model and a Method. Journal of Experimental Psychology: Human Perception and Performance, 10(2), 276-291.

Los, S. A. (2004). Inhibition of return and nonspecific preparation: separable inhibitory control mechanisms in space and time. Perception \& Psy- 
chophysics, 66(1), 119-130. https://doi.org/10.3758/BF03194866

Los, S. A. (2013). The role of response inhibition in temporal preparation: Evidence from a go/no-go task. Cognition, 129(2), 328-344. https://doi.org/10.1016/j.cognition.2013.07.013

Los, S. A., Kruijne, W., \& Meeter, M. (2014). Outlines of a multiple trace theory of temporal preparation. Frontiers in Psychology, 5(SEP). https://doi.org/10.3389/fpsyg.2014.01058

Menceloglu, M., Grabowecky, M., \& Suzuki, S. (2017). Comparing the effects of implicit and explicit temporal expectation on choice response time and response conflict. Attention, Perception, \& Psychophysics, 79(1), 169-179. https://doi.org/10.3758/s13414-016-1230-4

Martens, S., \& Johnson, A. (2005). Timing attention: Cuing target onset interval attentional blink. Memory \& Cognition, 33(2), 234-240. Retrieved from https://link.springer.com/content/pdf/10.3758

Miniussi, C., Wilding, E. L., Coull, J. T., \& Nobre, A. C. (1999). Orienting attention in time. Modulation of brain potentials. Brain, 122(8), 1507-1518. https://doi.org/10.1093/brain/122.8.1507

Mostofsky, S., \& Simmonds, D. (2008). Response inhibition and response selection: two sides of the same coin. Journal of Cognitive Neuroscience, 20(5), 751-761. https://doi.org/10.1162/jocn.2008.20500

Niemi, P., \& Naatanen, R. (1981). FP and Simple Reaction Time. Psychological Bulletin, 89(1), 133-162.

Nieuwenhuis, S., \& de Kleijn, R. (2013). The impact of alertness on cognitive control. Journal of Experimental Psychology: Human Perception and Performance, 39(6), 1797-1801. https://doi.org/10.1037/a0033980

Nobre, A. C. (2001). Orienting attention to instants in time. Neuropsychologia, 39(12), 1317-1328 . https://doi.org/10.1016/S0028-3932(01)00120-8

Pratte, M. S., Rouder, J. N., Morey, R. D., \& Feng, C. (2010). Exploring the differences in distributional properties between Stroop and Simon effects using delta plots. Attention, Perception \& Psychophysics, 72(7), 2013-2025. https://doi.org/10.3758/APP.72.7.2013

Ratcliff, R. (1979). Group Reaction Time Distributions and an Analysis of Distribution Statistics. Psychological Bulletin, 86(3), 446-461. Retrieved from http://psycnet.apa.org/fulltext/1979-27730-001.pdf

Ridderinkhof, K. R. (2002). Activation and suppression in conflict tasks: empirical clarification through distributional analyses. In W. Prinz \& B. Hommel (Eds.),. Common Mechanisms in Perception and Action. Attention \&. Performance, Vol. XIX (pp. 494-519). Oxford: Oxford. University Press.

Ridderinkhof, K. R., van den Wildenberg, W. P. M., Wijnen, J., \& Burle, B. (2004). Response inhibition in conflict tasks is revealed in delta plots. In M. I. Posner (Ed.), Cognitive Neuroscience of Attention (pp. 369-377). New York: Guilford Press.

Ridderinkhof, K., Forstmann, B. U., Wylie, S. A., Burle, B., \& van den Wildenberg, W. P. M. (2011). Neurocognitive mechanisms of action control: Resisting the call of the Sirens. Wiley Interdisciplinary Reviews: Cognitive Science, 2(2), 174-192. https://doi.org/10.1002/wcs.99

Rohenkohl, G., Gould, I. C., Pessoa, J., \& Nobre, A. C. (2014). Combining spatial and temporal expectations to improve visual perception. Journal of Vision, 14(4), 8-8. https://doi.org/10.1167/14.4.8 Rolke, B. (2008). Temporal preparation facilitates perceptual identification of letters. Perception and Psychophysics, 70(7), 1305-1313. https://doi.org/10.3758/PP.70.7.1305

Rolke, B., \& Hofmann, P. (2007). Temporal uncertainty degrades perceptual processing. Psychonomic Bulletin and Review, 14(3), 522-526. https://doi.org/10.3758/BF03194101

Seibold, V. C., \& Rolke, B. (2014a). Does temporal preparation facilitate visual processing in a selective manner? Evidence from attentional capture. Acta Psychologica, 151, 51-61. https://doi.org/10.1016/j.actpsy.2014.05.012

Seibold, V. C., \& Rolke, B. (2014b). Does temporal preparation speed up visual processing? Evidence from the N2pc. Psychophysiology, 51(6), 529-538. https://doi.org/10.1111/psyp. 12196
Simon, J. R. (1969). Reactions toward the source of stimulation. Journal of Experimental Psychology, 81(1), 174-176. http://dx.doi.org/10.1037/h0027448

Simon, J. R. (1990). The effect of an irrelevant directional cue on human information processing. In R. Proctor \& T. Reeve (Eds.), Stimulus-Response compatibility: An integrated perspective (pp. 31-88). Amsterdam: NorthHolland.

Stroop, J. (1935). Studies of interference in serial verbal reactions. Journal of Experimental Psychology, 18(6), 643-662. doi:10.1037/ h0054651

Tandonnet, C., Davranche, K., Meynier, C., Burle, B., Vidal, F., \& Hasbroucq, T. (2012). How does temporal preparation speed up response implementation in choice tasks? Evidence for an early cortical activation. Psychophysiology, 49(2), 252-260. https://doi.org/10.1111/j.1469-8986.2011.01301.x

Touge, T., Taylor, J. L., \& Rothwell, J. C. (1998). Reduced excitability of the cortico-spinal system during the warning period of a reaction time task. Electroencephalography and Clinical Neurophysiology - Electromyography and Motor Control, 109(6), 489-495. https://doi.org/10.1016/S0924980X(98)00050-2

Vallesi, A., Arbula, S., \& Bernardis, P. (2014). Functional dissociations in temporal preparation: Evidence from dual-task performance. Cognition, 130(2), 141-151. https://doi.org/10.1016/j.cognition.2013.10.006

Vallesi, A., \& Shallice, T. (2007). Developmental Dissociations of Preparation Over Time: Deconstructing the Variable FP Phenomena. Journal of Experimental Psychology: Human Perception and Performance, 33(6), 1377-1388. https://doi.org/10.1037/0096-1523.33.6.1377

van den Wildenberg, W. P. M., Wylie, S. A., Forstmann, B. U., Burle, B., Hasbroucq, T., \& Ridderinkhof, K. R. (2010). To Head or to Heed? Beyond the Surface of Selective Action Inhibition: A Review. Frontiers in Human Neuroscience, 4, 222. https://doi.org/10.3389/fnhum.2010.00222

Vangkilde, S., Coull, J. T., \& Bundesen, C. (2012). Great expectations: Temporal expectation modulates perceptual processing speed. Journal of Experimental Psychology: Human Perception and Performance, 38(5), 1183-1191. https://doi.org/10.1037/a0026343

Verbruggen, F., \& Logan, G. D. (2008). Automatic and controlled response inhibition: Associative learning in the go/no-go and stop-signal paradigms. Journal of Experimental Psychology: General, 137(4), 649-672. https://doi.org/10.1037/a0013170

Verbruggen, F., Logan, G. D., \& Stevens, M. A. (2008). STOP-IT: Windows executable software for the stop-signal paradigm. Behavior Research Methods, 40(2), 479-483. https://doi.org/10.3758/BRM.40.2.479

Vincent, S.B. (1912). The function of vibrissae in the behavior of the white rat. Behavior Monographs, 1(5), 1-82.

Visser, T. A. W. (2014). Evidence for deficits in the temporal attention span of poor readers. PLoS ONE, 9(3), e91278. https://doi.org/10.1371/journal.pone.0091278

Vohs, K.D., \& Baumeister, R.F. (2004). Handbook of Self- Regulation: Research, Theory, and Applications. New York, NY: Guilford Press.

Weinbach, N., \& Henik, A. (2012a). Temporal orienting and alerting - the same or different? Frontiers in Psychology, 3(236), 1-3. https://doi.org/10.3389/fpsyg.2012.00236

Weinbach, N., \& Henik, A. (2012b). The relationship between alertness and executive control. Journal of Experimental Psychology: Human Perception and Performance, 38(6), 1530-1540. https://doi.org/10.1037/a0027875

Weinbach, N., \& Henik, A. (2013). The interaction between alerting and executive control: Dissociating phasic arousal and temporal expectancy. Attention, Perception, \& Psychophysics, 75(7), 1374-1381. https://doi.org/10.3758/s13414-013-0501-6

Weinbach, N., Kalanthroff, E., Avnit, A., \& Henik, A. (2015). Can arousal modulate response inhibition? Journal of Experimental Psychology: Learning, Memory, and Cognition, 41(6), 1873-1877. https://doi.org/10.1037/xlm0000118 